\title{
ARRANGEMENTS, KZ SYSTEMS AND LIE ALGEBRA HOMOLOGY
}

\author{
EDUARD LOOIJENGA
}

To Terry Wall, for his 60th birthday.

\section{INTRODUCTION}

The Knizhnik-Zamolodchikov equations were originally defined in terms of a local system associated to tuples of finite dimensional irreducible representations of $\mathrm{SU}_{2}$, but were soon afterwards generalized to a Kac-Moody setting. The natural question that arises is whether these local systems admit a topological interpretation. A paper by Varchenko-Schechtman [8] comes close to answering this affirmatively and it is this article and related work that we intend to survey here.

Our presentation deviates at certain points from the original sources. First, we felt it worthwhile to introduce the notion of a Knizhnik-Zamolodchikov system (in (1.4)), whose value is enhanced by the simple criterion (1.5). Such systems also occur in the theory of root systems (both in a linear and in an exponential setting) and we took the occasion to discuss them briefly from our point of view. A KZ system leads to what is perhaps the most natural class of local systems on hyperplane complements endowed with a given extension over the whole space as vector bundle. That may already be sufficient reason for this notion to merit a more thorough investigation than we can give here.

Second, we treated the cohomology of hyperplane complements (due Arnol'd, Brieskorn and Orlik-Solomon) using the methods of sheaf theory, an approach we advocated on an earlier occasion for its effectiveness. Furthermore, we kept the statements free of genericity assumptions regarding the exponents; this applies in particular to the discussion in section 4. Finally, we avoided the use of Lie bialgebras structures and comodules in [8].

This is a slightly edited version of a manuscript written in the Spring of 1994. It is based on two talks given one year earlier in a seminar on hypergeometric functions in Utrecht.

\section{KNIZHNIK-ZAMOLODCHIKOV SYSTEMS}

(1.1) Let $U$ be a connected analytic manifold and $F$ a complex vector space. The trivial vector bundle $F \times U \rightarrow U$ has $\mathcal{F}:=\mathcal{O}_{U} \otimes F$ as its sheaf of holomorphic 
sections. A holomorphic connection on this vector bundle is given by an $\operatorname{End}(F)$ valued holomorphic 1-form $E=\sum_{i} \omega_{i} \otimes E_{i} \in \Omega^{1}(U) \otimes \operatorname{End}(F)$ :

$$
D_{E}: \mathcal{O}_{U} \otimes F \rightarrow \Omega_{U}^{1} \otimes F, \quad \phi \otimes v \mapsto d \phi \otimes v+\sum_{i} \phi \omega_{i} \otimes E_{i}(v)
$$

It is customary to extend $D_{E}$ to a derivation of degree 1 in $\Omega_{U}^{\bullet} \otimes F$ by

$$
D_{E}: \Omega_{U}^{k} \otimes F \rightarrow \Omega_{U}^{k+1} \otimes F, \quad \omega \otimes v \mapsto d \omega \otimes v+\sum_{i} \omega_{i} \wedge \omega \otimes E_{i}(v) .
$$

Then

$$
D_{E} D_{E}(\omega \otimes v)=\sum_{i} d \omega_{i} \wedge \omega \otimes E_{i}(v)+\sum_{i, j} \omega_{i} \wedge \omega_{j} \wedge \omega \otimes E_{i} E_{j}(v)
$$

So $D_{E} D_{E}$ is $\mathcal{O}_{U}$-linear and is equal to wedging with $d E+E \wedge E \in \Omega^{2}(U) \otimes \operatorname{End}(F)$. The last expression is called the curvature of the connection $D_{E}$. If it is constant zero, we say that $D_{E}$ is flat; then the local sections of the vector bundle annihilated by $D_{E}$ define a local system $\mathbb{F} \subset \mathcal{O}_{U} \otimes F$ and $\left(\Omega_{U}^{\bullet}, D_{E}\right)$ becomes a complex of coherent sheaves which resolves $\mathbb{F}$; this is the De Rham complex of $\mathbb{F}$.

In case $U$ is an algebraic variety there is defined a special class of holomorphic differentials on $U$, the logarithmic forms: If $\bar{U} \supset U$ is a smooth completion of $U$ which adds to $U$ a normal crossing divisor $D$, then a regular differential $\omega$ on $U$ is said to be logarithmic if it defines a section of $\Omega_{\bar{U}}(\log D)$; it is well-known that this property is independent of the choice of the completion. According to Deligne [3] such a differential is always closed. If instead of being a normal crossing divisor, $D$ is arrangement-like, that is, in local-analytic coordinates given by a product of linear forms $f_{1}, \ldots, f_{k}$, then a regular differential on $U$ is logarithmic if and only if in any such coordinate patch it is locally a linear combination combination of the logarithmic forms $f_{1}^{-1} d f_{1}, \ldots, f_{k}^{-1} d f_{k}$ with analytic coefficients.

We say that the connection $E$ (on the trivial vector bundle over $U$ with fiber $F$ ) is logarithmic if its coefficients are. For such connections there is the following flatness criterion:

(1.2) Proposition. Suppose that $\bar{U} \supset U$ is a smooth completion of $U$ which adds to $U$ an arrangement-like divisor $D$ whose irreducible components $D_{\alpha}$ are smooth. Suppose that $\bar{U}$ has no nonzero regular 2 -forms and that and any irreducible component $D_{\alpha}$ has no nonzero regular 1 -forms. Then a logarithmic connection $E$ on $U$ is flat if and only if for every for every intersection $I$ of two distinct irreducible components of $D$, the sum $\sum_{D_{\alpha} \supset I} \operatorname{Res}_{D_{\alpha}} E$ commutes with each of its terms $\operatorname{Res}_{D_{\alpha}} E$ $\left(D_{\alpha} \supset I\right)$.

Proof. A straightforward local calculation shows that the last property is equivalent to the vanishing of any double residue of $E \wedge E$ along any $I$. So it is certainly a necessary condition for flatness, but it is also sufficient: if this is the case for every $I$, then the residue of $E \wedge E$ along any $D_{\alpha}$ has as coefficients regular differentials on $D_{\alpha}$. Since there are no nonzero everywhere regular differentials on any $D_{\alpha}$ it 
follows that $E \wedge E$ is regular everywhere. As there are no nonzero regular 2-forms on $\bar{U}$, we have in fact that $E \wedge E=0$.

We remark that if we add to a flat logarithmic connection a logarithmic differential (which acts on $\mathcal{O}_{U} \otimes F$ via the first factor, then the resulting connection is still logarithmic and flat.

The examples we shall deal with involve a projective space $P$ and a finite collection $\mathcal{C}$ of hyperplanes in $P$. Our base manifold $U$ is then $P-\cup_{H \in \mathcal{C}} H$. From (1.2) we immediately conclude:

(1.3) Corollary. Let $E$ define a logarithmic connection $D_{E}$ on $\mathcal{O}_{U} \otimes F$. Then this connection is flat if and only if for every codimension two intersection $I$ of members of $\mathcal{C}$, the sum $\sum_{H \in \mathcal{C}, H \supset I} \operatorname{Res}_{H} E$ commutes with each of its terms $\operatorname{Res}_{H} E$ $(H \in \mathcal{C}, H \supset I)$.

(1.4) If the equivalent conditions of the corollary are fulfilled we say that the quadruple $(P, \mathcal{C}, F, E)$ (or simply $E$ ) defines a Knizhnik-Zamolodchikov (KZ) system. A flat local section of a $\mathrm{KZ}$ system $(F, E)$ is given by an $F$-valued holomorphic map on an open subset of $U$; the composite of such a map with a linear form on $F$ is called a hypergeometric function.

Usually a KZ system will be given in terms of an affine space $V$ and a finite collection $\mathcal{C}$ of hyperplanes in $V$ with $U=V-\cup_{H \in \mathcal{C}} H$. Then by adding to $V$ the hyperplane at infinity we find ourselves in the previous case. Every $H \in \mathcal{C}$ determines a logarithmic differential

$$
\omega_{H}:=\frac{d f_{H}}{f_{H}},
$$

where $f_{H}$ is an affine-linear form whose zero set is $H$. This is a closed holomorphic form on $U$ which does not depend on the choice of $f_{H}$. The $\omega_{H}$ 's make up a basis of the logarithmic differentials on $U$, so that any logarithmic connection on $\mathcal{O}_{U} \otimes F$ is of the form $E=\sum_{H \in \mathcal{C}} \omega_{H} \otimes E_{H}$ with $E_{H} \in \operatorname{End}(F)$. So:

(1.5) Corollary. The connection $D_{E}$ is flat if and only if for every codimension two intersection $I$ of members of $\mathcal{C}$, the sum $\sum_{H \in \mathcal{C}, H \supset I} E_{H}$ commutes with each of its terms $E_{H}(H \in \mathcal{C}, H \supset I)$.

(1.6) Here are two important classes of examples.

1. (Heckman-Opdam-Dunkl) Let $V$ and $\mathcal{C}$ be as above and let $G$ be a finite subgroup of $G L(V)$ which preserves $\mathcal{C}$. (A case in point is when $G$ is a finite complex reflection group and $\mathcal{C}$ is the collection of fixed point hyperplanes of complex reflections in $G$.) We take for $F$ the group algebra $\mathbb{C}[G]$ of $G$ (acting on itself by left multiplication) and for every $H \in \mathcal{C}$ we choose $E_{H} \in \mathbb{C}\left[Z_{G}(H)\right]$ (where $Z_{G}(H)$ is the group of $g \in G$ which fix $H$ pointwise) such that $E_{g(H)}=g E_{H} g^{-1}$ for all $H \in \mathcal{C}$ and $g \in G$. It is clear that then for a codimension two intersection $I$ of reflection hyperplanes the element $\sum_{H \in \mathcal{C}, H \supset I} E_{H}$ is central in $\mathbb{C}\left[Z_{G}(I)\right]$, so that $E:=\sum_{H} \omega_{H} \otimes E_{H}$ defines a KZ system.

The monodromy group of this local system will take values in the group of units of $\mathbb{C}[G]$. So if $F$ is a finite dimensional complex representation of $G$, then $F$ together 
with the image of $E$ in $\Omega_{U} \otimes \operatorname{End}(F)$ defines another such system. Notice that these local systems come with a natural $G$-action and therefore descend to local systems on a Zariski open subset of the $G$-orbit space of $V$.

2. Let $\mathfrak{g}$ be a finite dimensional complex Lie algebra and $B$ a nondegenerate symmetric bilinear form on $\mathfrak{g}$. (Such a form exists if and only if $\mathfrak{g}$ is reductive.) Let $B^{\vee} \in \mathfrak{g} \otimes \mathfrak{g}$ represent the inverse form on the dual of $\mathfrak{g}$ (so if $\left(e_{k}\right)_{k}$ is an orthonormal basis of $\mathfrak{g}$, then $\left.B^{\vee}=\sum_{k} e_{k} \otimes e_{k}\right)$. The Lie bracket is defined by a map $\mathfrak{g} \otimes \mathfrak{g} \rightarrow \mathfrak{g}$. If we denote its adjoint by $u: \mathfrak{g} \rightarrow \mathfrak{g} \otimes \mathfrak{g}$, then we have the following identities in $U \mathfrak{g} \otimes U \mathfrak{g}:$

$$
-\left[X \otimes 1, B^{\vee}\right]=\left[1 \otimes X, B^{\vee}\right]=u(X) .
$$

Let for $1 \leq k, l \leq 3, k \neq l, E_{k, l} \in \mathfrak{g}^{\otimes 3}$ be obtained by inserting $B^{\vee}$ in the slots $k$ and $l$. The $\mathfrak{g}$-invariance of $B$ amounts to the property that the expression $B(X,[Y, Z])$ is anti-symmetric in its three arguments. If we dualize, we find that $\left[E_{\sigma(1), \sigma(2)}, E_{\sigma(2), \sigma(3)}\right]$ is anti-symmetric in $\sigma \in \mathfrak{S}_{3}$. This implies that the sum $E_{1,2}+E_{2,3}+E_{1,3}$ commutes with each of its terms.

Now let for $n \geq 2, V_{n}$ be the subspace of $\mathbb{C}^{n}$ defined by $z_{1}+\cdots+z_{n}=0, \mathcal{C}_{n}$ the collection hyperplanes $z_{k}-z_{l}=0(1 \leq k<l \leq n)$ and $U_{n}$ the complement in $V_{n}$ of the union of these. Denote the logarithmic form associated to $z_{k}-z_{l}=0$ by $\omega_{k, l}$ and let $E_{k, l} \in U \mathfrak{g}^{\otimes n}$ have the obvious meaning. Then it follows from the preceding remarks that $E:=\sum_{1<k<l<n} \omega_{k, l} \otimes E_{k, l} \in B_{U_{n}}^{\vee} \otimes U \mathfrak{g}^{\otimes n}$ defines a KZ system. If $M_{1}, \ldots, M_{n}$ are representations of $\mathfrak{g}$, then the same is true for the image of $E$ in $B_{U}^{\vee} \otimes \operatorname{End}\left(M_{1} \otimes \cdots \otimes M_{n}\right)$. The equations defining the flat sections of such systems are known as the Knizhnik-Zamolodchikov equations.

Since $B^{\vee}$ is a $\mathfrak{g}$-invariant element (of $\mathfrak{g}^{\otimes 2}$ ), so are the $E_{k, l}$ 's. This implies that the $\mathfrak{g}$-action on the fibres sends flat sections to flat sections. So if

$$
M_{1} \otimes \cdots \otimes M_{n}=\oplus_{S} F_{S} \otimes S
$$

is a decomposition into isotypical components (with $S$ running over a system of mutually inequivalent irreducible representations of $\mathfrak{g})$, then by Schur's lemma the connection actually takes values in $\oplus_{S} \operatorname{End}\left(F_{S}\right)$. Hence for each $S$, we have a $\mathrm{KZ}$ system on the trivial vector bundle with fiber $F_{S}=\operatorname{Hom}_{\mathfrak{g}}\left(S, M_{1} \otimes \cdots \otimes M_{n}\right)$.

An interesting case is $\mathfrak{g}=\mathfrak{g l}(W)$ (where $W$ is a finite dimensional vector space) and $M_{i}=W$ for all $i$. A classical theorem of Weyl asserts that $W^{\otimes n}$ decomposes as a direct sum of irreducible $G L(W) \times \mathfrak{S}_{n}$-modules with multiplicity one. This implies that each $F_{S}$ is an irreducible $\mathfrak{S}_{n}$-module. It can be shown that its $\mathrm{KZ}$ system is of the type discussed in example 1.

\section{INTERMEZZO: THE TORIC LOGARITHMIC SYSTEM ASSOCIATED TO A ROOT SYSTEM}

(2.1) There is also a toric version of the $\mathrm{KZ}$ connection, which is due to Cherednik and Matsuo. Although this will not play a role here we cannot resist the temptation to give it a similar treatment. Let $R$ be a finite, possibly nonreduced root system in a real vector space $V$ with Weyl group $W$ and $L \subset V$ a $W$-invariant lattice containing the roots. Then $T:=\operatorname{Hom}\left(L, \mathbb{C}^{*}\right)$ is an algebraic torus with $W$-action. Every $\alpha \in R$ determines a character $\chi_{\alpha}: T \rightarrow \mathbb{C}^{*}$. We denote the 
kernel of this character by $D_{\alpha}$. Our logarithmic connection will be defined on $U:=T-\cup_{\alpha \in R} D_{\alpha}$ and will have values in $\mathbb{C}[W]$. In order to be able to apply (1.2) we need a completion of $T$. We take (as the most natural choice) the torus embedding $T \subset \hat{T}$ defined by the decomposition of $\operatorname{Hom}\left(\mathbb{C}^{*}, T\right) \otimes_{\mathbb{Z}} \mathbb{R} \cong V^{\vee}$ into chambers. This completion need not be smooth, but it admits a smooth Galois cover and that is good enough for our purpose. It adds to $T$ a normal crossing divisor (or rather an orbit space of such a divisor under a finite group action) whose irreducible components are bijectively indexed by the one-dimensional facets in $V^{\vee}$. Every one-dimensional facet contains a unique indivisible element of $L$, so if $\Pi \subset L$ denotes the collection of such elements, then for every $p \in \Pi$ we have an irreducible component $D^{p}$ of $\hat{L}-L$. Then $D_{\alpha}, \alpha \in R$ meets $D^{p}, p \in \Pi$ if and only if $\alpha(p)=0$ and in that case no other irreducible component of $\hat{T}-U$ passes through $D_{\alpha} \cap D^{p}$. For every root $\alpha \in R$ we define two logarithmic differentials on $U$ :

$$
\eta_{\alpha}:=\chi_{\alpha}^{*}\left(\frac{d t}{t}\right) \quad, \quad \omega_{\alpha}:=\chi_{\alpha}^{*}\left(\frac{(t+1) d t}{(t-1) t}\right) .
$$

A simple calculation shows that these elements have the following residues:

$$
\operatorname{Res}_{D^{p}} \eta_{\alpha}=\alpha(p), \quad \operatorname{Res}_{D^{p}} \omega_{\alpha}=-|\alpha(p)|, \quad \operatorname{Res}_{D_{\alpha}} \omega_{\alpha}=2
$$

all other residues vanish. We fix a chamber $C$ in $V^{\vee}$ so that we have system of positive roots $R^{+}$and we define for $\alpha \in R, \epsilon_{\alpha} \in$ End $\mathbb{C}[W]$ by

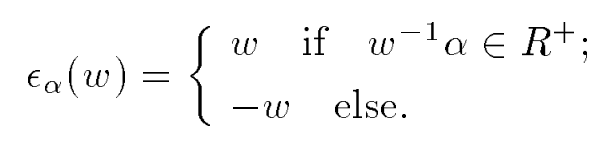

These endomorphisms possess the following properties:

(i) $\epsilon_{\alpha}+\epsilon_{-\alpha}=0$,

(ii) for $w \in W, w \epsilon_{\alpha} w^{-1}=\epsilon_{w(\alpha)}$.

(2.2) Proposition. If $k \in R \rightarrow k_{\alpha} \in \mathbb{C}$ is a $W$-invariant function, then

$$
E:=\sum_{\alpha \in R} k_{\alpha}\left(\omega_{\alpha} \otimes s_{\alpha}+\eta_{\alpha} \otimes s_{\alpha} \epsilon_{\alpha}\right)
$$

defines a flat logarithmic $W$-equivariant connection on the trivial bundle over $U$ with fiber $\mathbb{C}[W]$.

Proof. The $W$-invariance is clear from property (ii) above. Since $\hat{T}$ is rational, it has no regular 2 -form. For the same reason, no irreducible component of $\hat{T}-U$ has a regular 1 -form. We verify the criterion (1.2) along every intersection $I$ of two distinct irreducible components of $\hat{T}-U$. First note that

$$
\begin{aligned}
& \operatorname{Res}_{D_{\alpha}} E=2 k_{\alpha} s_{\alpha}, \\
& \operatorname{Res}_{D^{p}} E=\sum_{\beta \in R} k_{\beta}\left(-|\beta(p)| s_{\beta}+\beta(p) s_{\beta} \epsilon_{\beta}\right) .
\end{aligned}
$$


If $I$ is of the form $D_{\alpha} \cap D_{\beta}$, then the argument of example 1 applies. If $I$ is of the form $D_{\alpha} \cap D^{p}$, then we need to show that $\operatorname{Res}_{D_{\alpha}} E=s_{\alpha}$ commutes with $\operatorname{Res}_{D^{p}} E$. But this follows from the $W$-invariance of $E$ and the fact that $s_{\alpha}(p)=p$. There remains the case when $I$ is of the form $D^{p} \cap D^{p^{\prime}}$. This intersection is nonempty only if $p$ and $p^{\prime}$ are in the closure of a single chamber; since $E$ is $W$-invariant, we may as well assume that this chamber is $C$. Then

$$
\operatorname{Res}_{D^{p}} E=\sum_{\beta \in R^{+}}-2 k_{\beta} \beta(p) s_{\beta}\left(1-\epsilon_{\beta}\right) .
$$

and a similar expression for $\operatorname{Res}_{D^{p^{\prime}}} E$. Now, if $\beta$ is a positive root, then $(1-$ $\left.\epsilon_{\beta}\right)(w)=0$ unless $w^{-1} \beta \in R^{-}$. We can express this in a better way if we write $w \in W$ as the reduced expression in the simple reflections $w=s_{\alpha_{1}} s_{\alpha_{2}} \cdots s_{\alpha_{l}}$, where $\alpha_{1}, \ldots, \alpha_{l}$ are simple roots relative $C$. Then $\left(1-\epsilon_{\beta}\right)(w)=0$ unless there is a (unique) $i \in\{1, \ldots, l\}$ with $\beta$ a positive mutiple of $s_{\alpha_{1}} \ldots s_{\alpha_{i-1}}\left(\alpha_{i}\right)$ and in that case $\left(1-\epsilon_{\beta}\right)(w)=2 s_{\alpha_{1}} \cdots \widehat{s_{\alpha_{i}}} \cdots s_{\alpha_{l}}$. Hence

$$
\operatorname{Res}_{D^{p}} E(w)=\sum_{i=1}^{l}-4 \tilde{k}_{\alpha_{i}} s_{\alpha_{1}} \ldots s_{\alpha_{i-1}}\left(\alpha_{i}\right)(p) s_{\alpha_{1}} \ldots \widehat{s_{\alpha_{i}}} \cdots s_{\alpha_{l}} .
$$

where $\tilde{k}_{\alpha_{i}}$ denotes the sum of the $k_{\beta}$ 's for which $\beta$ is a positive multiple of $\alpha_{i}$. This formula gives us for the commutator $\left[\operatorname{Res}_{D^{p^{\prime}}} E, \operatorname{Res}_{D^{p}} E\right.$ ] applied to $w$ a linear combination of the elements $s_{\alpha_{1}} \cdots \widehat{s_{\alpha_{i}}} \cdots \widehat{s_{\alpha_{j}}} \cdots s_{\alpha_{k}}$; the coefficient of that element is proportional to the value in $\left(p, p^{\prime}\right)$ of the 2 -form

$$
s_{\alpha_{1}} \cdots s_{\alpha_{i-1}}\left(\alpha_{i} \wedge\left(1-s_{\alpha_{i}}\right) s_{\alpha_{i+1}} \cdots s_{\alpha_{j-1}} \alpha_{j}\right) .
$$

Since the image of $1-s_{\alpha_{i}}$ is spanned by $\alpha_{i}$, this 2 -form is clearly zero. Hence Res $D^{p}$ and $\operatorname{Res}_{D^{p^{\prime}}}$ commute. This completes the proof.

(2.3) Remarks. 1. Matsuo actually proves the flatness of a more general connection: he allows an additional term in $\Omega(T) \otimes \operatorname{End} \mathbb{C}[W]=\operatorname{Hom}(\mathbb{C}[W], \Omega(T) \otimes \mathbb{C}[W])$ which depends on a choice of $\lambda \in V \otimes \mathbb{C}$ : if $d \lambda$ is the translation invariant differential on $T$ corresponding to $\lambda$, then it is given by $w \in W \mapsto w d \lambda \otimes w \in \Omega(T) \otimes \mathbb{C}[W]$. In a sense this generalization is only apparent, for on the universal cover $\operatorname{Hom}_{\mathbb{R}}(V, \mathbb{C})$ of $T$ this new connection is equivalent to the old one by means of the endomorphism of $\mathcal{O}_{T} \otimes \mathbb{C}[W]$ which multiplies $w$ by $w \lambda$.

2. Since the algebra $\mathbb{C}[W]$ does not contain the endomorphims $\epsilon_{\alpha}$, the monodromomy group will no longer take values in the group of units of $\mathbb{C}[W]$. So there is no obvious way to descend the connection to any representation of $W$. Heckman and Opdam have however identified the monodromy group in terms of an affine Hecke algebra and so one expects the connection to descend to certain representations of this algebra.

\section{Cohomology of arRangements}

(3.1) Let $V$ be a finite dimensional affine space over the complex numbers and let $\mathcal{C}$ be a collection of affine hyperplanes in $V$. We denote

$$
D:=\cup_{H \in \mathcal{C}} H, \quad U:=V-D
$$


and name their inclusions in $V i: D \subset V$ and $j: U \subset V$. A facet of $\mathcal{C}$ is a nonempty intersection of members of $\mathcal{C}$ (this includes $V$ ); the collection of codimension $p$-facets is denoted by $\mathcal{C}^{p}$. We give a resolution $\mathcal{F}^{\bullet}$ of the sheaf $i_{*} \mathbb{Z}_{D}$ which generalizes a standard construction in the normal crossing case: denote by $D^{[p]}$ the disjoint union of the $p$-facets of $\mathcal{C}$, by $i_{p}: D^{[p]} \rightarrow V$ be the obvious map and by $U^{[p]}$ the difference of $i_{p}\left(D^{[p]}\right)$ and $i_{p}\left(D^{[p+1]}\right)$ (but we regard $U^{[p]}$ also as a subset of $D^{[p]}$ ). Let $\mathcal{F}^{1}:=\mathbb{Z}_{D^{[1]}}$. Then we have an inclusion $i_{*} \mathbb{Z}_{D} \subset i_{1 *} \mathcal{F}^{1}$ whose cokernel $\mathcal{F}^{2}$ is supported by the image of $D^{[2]}$. This cokernel is constant on each connected component of $U^{[2]}$ and so the direct image of $\mathcal{F}^{2} \mid U^{[2]}$ on $D^{[2]}$ of is sheaf $\mathcal{F}^{2}$ which is constant on every component $L$ of $D^{[2]}$. There is an obvious morphism $i_{1 *} \mathcal{F}^{1} \rightarrow i_{2 *} \mathcal{F}^{2}$ whose kernel is $i_{*} \mathbb{Z}_{D}$ and whose cokernel is supported by the image of $i_{3}$. It is clear that we can continue in this way.

Here is a more precise description. Define a $p$-flag of $\mathcal{C}$ as a chain

$$
L^{\bullet}=\left(L^{p} \subset L^{p-1} \subset \cdots \subset L^{0}=V\right), \quad p=0,1, \ldots
$$

with $L^{i} \in \mathcal{C}^{i}$. Let $F^{p}$ be the free abelian group generated by the $p$-flags of $\mathcal{C}$ modulo the relations

$$
\sum_{L \in \mathcal{C}^{i}, L^{i+1} \subset L \subset L^{i-1}}\left[L^{p} \subset \cdots \subset L^{i+1} \subset L \subset L^{i-1} \subset \cdots \subset L^{0}\right]=0, \quad 0<i<p .
$$

We turn this into a complex by putting

$$
d\left(\left[L^{p} \subset \cdots \subset L^{0}\right]\right):=\sum_{L \in \mathcal{C}^{p+1}, L \subset L^{p}}\left[L \subset L^{p} \subset \cdots \subset L^{0}\right] .
$$

If we restrict ourselves to those hyperplanes that contain a given $L \in \mathcal{C}^{p}$, then we shall write $F_{L}^{\bullet}$ instead. It is easy to see that the complex $\mathcal{F}^{p} \mid L$ is the constant sheaf $\mathbb{Z}_{L} \otimes F_{L}^{p}$

(3.2) Proposition. There is a natural isomorphism $H^{\bullet}(V, D ; \mathbb{Z}) \cong H^{\bullet}\left(F^{\bullet}, d\right)$.

Proof. If $\mathcal{I}$ is any sheaf on $V$, then there is a standard exact sequence

$$
0 \rightarrow j_{!} j^{*} \mathcal{I} \rightarrow I \rightarrow i_{*} i^{*} \mathcal{I} \rightarrow 0
$$

If $i^{*} \mathcal{I}$ has the property that every local section is a sum of local sections with support contained in a single member of $\mathcal{C}$, then we can use the resolution $\mathcal{F}^{\bullet}$ to resolve $i_{*} i^{*} \mathcal{I}$, and we get an exact complex

$$
0 \rightarrow j ! j^{*} \mathcal{I} \rightarrow \mathcal{I} \rightarrow i_{1 *}\left(i_{1}^{*} \mathcal{I} \otimes \mathcal{F}^{1}\right) \rightarrow i_{2 *}\left(i_{2}^{*} \mathcal{I} \otimes \mathcal{F}^{2}\right) \cdots
$$

If we regard this as a resolution of $j ! j^{*} \mathcal{I}$, then there is an associated spectral sequence

$$
E_{1}^{p, q}=H^{q}\left(D^{[p]}, i^{*} \mathcal{I} \otimes \mathcal{F}^{p}\right)=\bigoplus_{L \in \mathcal{C}^{p}} H^{q}(L ; \mathcal{I} \mid L) \otimes F_{L}^{p} \Rightarrow H^{p+q}\left(j ! j^{*} \mathcal{I}\right)
$$


where we convene that $i_{0}: D^{[0]}=V$ and $\mathcal{F}_{0}=\mathbb{Z}_{V}$. This applies in particular to the Godement resolution $\mathcal{I}^{\bullet}$ of the constant sheaf $\mathbb{Z}_{V}$. Then this spectral sequence becomes

$$
E_{1}^{p, q}=\bigoplus_{L \in \mathcal{C}^{p}} H^{q}(L ; \mathbb{Z}) \otimes F_{L}^{p} \Rightarrow H^{p+q}(V, D ; \mathbb{Z})
$$

Since $H^{q}(L ; \mathbb{Z})=0$ for $q \neq 0$, the spectral sequence degenerates at $E_{2}$ and the proposition follows.

We have also a dual use for the $F^{p}$ 's, which at the same time leads to a homological interpretation of $F_{L}^{p}$. Let $U_{L}$ denote the complement in $V$ of the union of hyperplanes in $\mathcal{C}$ that contain $L$.

(3.3) Proposition. There is a natural isomorphism $H^{p}(U ; \mathbb{Z}(p)) \cong\left(F^{p}\right)^{\vee}$. If $L \in \mathcal{C}^{p}$, then $\left(F^{p}\right)^{\vee}$ is naturally isomorphic to $H^{p}\left(U_{L} ; \mathbb{Z}(p)\right)$, so that the canonical map $H^{p}(U ; \mathbb{Z}) \rightarrow \oplus_{L \in \mathcal{C}^{p}} H^{p}\left(U_{L} ; \mathbb{Z}\right)$ is an isomorphism.

Proof. Let $\mathcal{I}^{\bullet}$ be the Godement resolution of $\mathbb{Z}_{V}$. Then a similar argument as in the proof of (3.2) shows that we have an exact complex of graded differential sheaves

$$
\cdots \rightarrow i_{2 *}\left(\operatorname { H o m } ( \mathcal { F } ^ { 2 } , i _ { 2 } ^ { ! } \mathcal { I } ^ { \bullet } ) \rightarrow i _ { 1 * } \left(\operatorname{Hom}\left(\mathcal{F}^{1}, i_{1}^{!} \mathcal{I}^{\bullet}\right) \rightarrow \mathcal{I}^{\bullet} \rightarrow j_{*} j^{*} \mathcal{I}^{\bullet} \rightarrow 0\right.\right.
$$

The Thom isomorphism shows that $i_{p}^{!} \mathcal{I} \cdot$ is quasi-isomorphic to the shifted (and twisted) constant sheaf $\mathbb{Z}_{D^{[p]}}[-2 p](-p)$. This gives the spectral sequence

$$
\begin{aligned}
E_{1}^{-p, q}=H^{-2 p+q}\left(D^{[p]}, \operatorname{Hom}\left(\mathcal{F}^{p}, \mathbb{Z}(-p)\right)=\right. \\
=\bigoplus_{L \in \mathcal{C}^{p}} \operatorname{Hom}\left(F_{L}^{p}, H^{-2 p+q}(L ; \mathbb{Z})(-p)\right) \Rightarrow H^{-p+q}(U ; \mathbb{Z}) .
\end{aligned}
$$

Since $E_{1}^{-p, q}=0$ for $-2 p+q \neq 0$, the spectral sequence degenerates at the $E_{1}$-term, and it follows that $H^{p}(U ; \mathbb{Z}) \cong \oplus \oplus_{L \in \mathcal{C}^{p}}\left(F_{L}^{p}\right)^{\vee}(-p)=\left(F^{p}\right)^{\vee}(-p)$. If we apply this to the collection of hyperplanes passing through $L \in \mathcal{C}^{p}$, we find that $H^{p}\left(U_{L} ; \mathbb{Z}\right) \cong$ $\left(F_{L}^{p}\right)^{\vee}(-p)$.

This proposition shows that the cohomology of $U$ is torsion free. By the universal coefficient theorem, the homology of $U$ is then also torsion free. It can be shown that each $F_{L}^{p}$ is torsion free, so that $F_{L}^{p} \cong H_{p}\left(U_{L} ; \mathbb{Z}(-p)\right.$ ).

(3.4) Just as in the normal crossing case, there is another description of $H^{p}(U)$ in terms of logarithmic differentials. It goes as follows. The logarithmic differential $\omega_{H}$ defined in (1.4) is a closed form on $U-H$ whose period over a circle generating $H_{1}(V-H ; \mathbb{Z})$ is equal to $\pm 2 \pi \sqrt{-1}$. Hence $\omega_{H}$ defines a generator of $H^{1}(V-$ $H, \mathbb{Z}(-1))$. Let $A^{\bullet}$ be the $\mathbb{Z}$-algebra of meromorphic forms generated by the $\omega_{H}$ 's. It consists of closed forms which are holomorphic on $U$ so that we have a natural algebra homomorphism

$$
A^{\bullet} \rightarrow \oplus_{p} H^{p}(U ; \mathbb{Z}(p)) .
$$

According to Brieskorn this is an isomorphism (we shall give the proof below). The two descriptions are related through an iterated residue map: any $p$-flag $L^{\bullet}$ defines 
a linear form $\operatorname{Res}_{L} \cdot$ on $A^{p}$ by taking iterated residues along the flag: for $p \geq 1$ we let

$$
\operatorname{Res}_{L} \bullet: \omega \in A^{p} \mapsto \operatorname{Res}_{L^{p}} \operatorname{Res}_{L^{p-1}} \cdots \operatorname{Res}_{L^{1}}(\omega) \in \mathbb{Z},
$$

and we convene that $\operatorname{Res}_{V}$ is the identity map of $\mathbb{Z}$. A straightforward calculation shows that this factors through a linear map

$$
\operatorname{Res}^{p}: A^{p} \rightarrow\left(F^{p}\right)^{\vee}
$$

Its composite with the isomorphism $\left(F^{p}\right)^{\vee} \cong H^{p}(U ; \mathbb{Z}(p))$ yields the map $A^{p} \rightarrow$ $H^{p}(U ; \mathbb{Z}(p))$ above.

(3.5) Proposition. For every $p$ the maps $A^{p} \rightarrow H^{p}(U ; \mathbb{Z}(p))$ and $\operatorname{Res}^{p}: A^{p} \rightarrow$ $\left(F^{p}\right)^{\vee}$ are isomorphisms.

Proof. The two assertions are equivalent so that it is enough to prove one of them. We proceed with induction on $\operatorname{dim} V$; so we assume that $\operatorname{dim} V \geq 1$ and the assertions proved for arrangements in affine spaces of smaller dimension.

For $L \in \mathcal{C}^{p}$, let $A_{L}^{\bullet}$ be the algebra associated to the collection of $H \in \mathcal{C}$ that contain $L$. So if $p=\operatorname{codim} L$, then $A_{L}^{p}$ is the subgroup of $A^{p}$ spanned by the $\omega_{H 1} \wedge \cdots \wedge \omega_{H_{p}}$ with $H_{1} \cap \cdots \cap H_{p}=L$. Clearly, $A^{p}$ is spanned by the $A_{L}^{p}$ with $L \in \mathcal{C}^{p}$. Since $A_{L}^{p}$ maps to $\left(F_{L}^{p}\right)^{\vee}$, it is enough to show that $A_{L}^{p} \rightarrow\left(F_{L}^{p}\right)^{\vee}$ is an isomorphism. This follows from our induction hypothesis unless $p=\operatorname{dim} V$. We therefore concentrate on the case that $p=\operatorname{dim} V$ and $\cap_{H \in \mathcal{C}} H$ is a singleton $\{0\}$. Choose $H \in \mathcal{C}$ and let $f_{H}: V \rightarrow \mathbb{C}$ be an affine-linear form whose zero set is $H$. Put $V_{1}:=f^{-1}(1)$. With an obvious interpretation of notation we have an isomorphism $U_{1} \times(\mathbb{C}-\{0\}) \rightarrow U,(z, t) \mapsto t z$. By inductive assumption, there are is a natural isomorphism $A_{1}^{p-1}=\left(F_{1}^{p}\right)^{\vee}$. We have an exact sequence

$$
0 \rightarrow F_{1}^{p} \rightarrow F^{p} \rightarrow F_{1}^{p-1} \rightarrow 0,
$$

where the last map assigns to a $p$-flag $\{0\}=L^{p} \subset \cdots \subset L^{0}$ the $(p-1)$-flag $L^{p-1} \cap V_{1} \subset \cdots L^{0} \cap V_{1}$. This parallels the sequence

$$
0 \rightarrow A_{1}^{p-1} \stackrel{\omega_{H} \wedge}{\longrightarrow} A^{p} \rightarrow A_{1}^{p} \rightarrow 0
$$

where the last map is given by restriction. It is a simple exercise to show that this sequence is exact also. There is an obvious morphism from this exact sequence to the dual of the previous one. By induction hypothesis this is an isomorphism on the extremal terms. The five lemma implies that we have an isomorphism on the middle term.

\section{TWISTED COEFFICIENTS}

If $(V, \mathcal{C}, F, E)$ defines a $\mathrm{KZ}$ system, then $A^{\bullet}(\mathcal{C}) \otimes F$ is a distinguished subcomplex of the De Rham complex of $\mathbb{F}$ with the differential $D_{E}$ operating as wedging with $E$. It is possible to give a topological interpretation of the cohomology of this subcomplex. As we will only need this when $F$ of dimension one, we shall restrict ourselves to that case. In that case $E$ is given by a logarithmic differential for which we shall write $\eta$ instead. 
It will be best to work on the projective completion of $V$ and so we may as well start out this way: until further notice $P$ is a complex projective space, $\mathcal{C}$ a finite collection of hyperplanes of $P, D$ the union of the hyperplanes $H \in \mathcal{C}, U:=P-D$, $F$ a one-dimensional complex vector space and $\eta$ a logarithmic differential which is regular on $U$. The the associated rank one local system on $U=P-D$ is denoted by $\mathbb{F}_{\eta}$.

(4.1) There is a natural way to blow up $P$ such that $D$ transforms into a normal crossing divisor. Let us say that a facet $L$ of $\mathcal{C}$ is abnormal if the projective space of hyperplanes containing $L$ has a projective basis of $(1+\operatorname{codim} L)$ members of $\mathcal{C}$. It is clear that the set of abnormal subspaces is closed under intersection. Such subspaces are the centers of a blow-up $\pi: \tilde{P} \rightarrow P$ which has the property that the pre-image of $D$ is a normal crossing divisor: blow up first the abnormal points; then the proper transforms of the abnormal lines become separated, blow these up and notice that the abnormal planes get separated and continue in this way. We end up with a blow-up $\pi: \tilde{P} \rightarrow P$ that resolves $D$ to a normal crossing divisor $\tilde{D}$. The following proposition is implicit in a paper by Esnault-Schechtman-Viehweg [4].

(4.2) Proposition. Let $\Phi(\eta)$ be the family of the sets $Z \subset U$ that are closed in $U$ and have the property that their closure in $P$ is disjoint from any abnormal facet $L$ with the property that $\sum_{H \supset L ; H \in \mathcal{C}} \operatorname{Res}_{H} \eta$ is a positive integer. This is a family of supports and there is natural isomorphism

$$
H^{\bullet}\left(A^{\bullet}, E \wedge\right) \rightarrow H_{\Phi(\eta)}^{\bullet}\left(V ; \mathbb{F}_{\eta}\right)
$$

Proof. Let $\Omega_{\tilde{P}}^{\bullet}(\log Y)$ be the logarithmic holomorphic De Rham complex of $(\tilde{P}, Y)$. This is a complex of free $\mathcal{O}_{\tilde{P}}$-modules which represents the total direct image of the sheaf of complex constants on $\tilde{P}-Y \cong U$. Notice that the elements of $A^{p}$ define global sections of this complex. Following Deligne [3], the spectral sequence defined by the logarithmic De Rham resolution

$$
E_{1}^{p, q}=H^{q}\left(\tilde{P}, \Omega_{\tilde{P}}^{p}(\log \tilde{D})\right) \Rightarrow H^{p+q}(U ; \mathbb{C})
$$

degenerates at the $E_{1}$-term. This means that we have a decreasing filtration $F^{\bullet}$ (the Hodge filtration) on $H^{\bullet}(U ; \mathbb{C})$ such that

$$
G r_{F}^{p} H^{n}(U ; \mathbb{C})=H^{n-p}\left(\tilde{P}, \Omega_{\tilde{P}}^{p}(\log \tilde{D})\right)
$$

By $(3.5), A^{p} \otimes \mathbb{C}$ maps isomorphically onto $H^{p}(U ; \mathbb{C})$. As $H^{0}\left(\tilde{P}, \Omega_{\tilde{P}}^{p}(\log \tilde{D})\right)=$ $H^{0}\left(P, \Omega_{P}^{p}(\log D)\right)=A^{p} \otimes \mathbb{C}$ it follows that $H^{q}\left(\tilde{P}, \Omega_{\tilde{P}}^{p}(\log \tilde{D})\right)=0$ for $q \neq 0$ and $H^{0}\left(\tilde{P}, \Omega_{\tilde{P}}^{p}(\log \tilde{D})\right)=A^{p} \otimes \mathbb{C}$. We now invoke another result of Deligne [1] which we state as follows:

Proposition. Let $X$ be a complex manifold, $Y \subset X$ a normal crossing divisor, and $\eta$ a closed global section of $\Omega_{X}^{1}(\log Y)$. Let $Y^{\prime} \subset Y$ be the union of irreducible components $Y_{i}$ of $Y$ for which $\operatorname{Res}_{Y_{i}} \eta$ is a positive integer and denote the inclusions 
$k: X-Y \rightarrow X-Y^{\prime}$ and $k^{\prime}: X-Y^{\prime} \subset X$. If $\mathbb{F}_{\eta}$ denotes the local system over $X-Y$ defined by the structure sheaf with flat connection $\eta$, then $\left(\Omega_{X}^{\bullet}(\log Y), d+\eta \wedge\right)$ represents $R^{\bullet} k_{!}^{\prime} R^{\bullet} k_{*} \mathbb{F}_{\eta}$.

This is not quite the way it is stated, but this is what is proved.

So if in our case, $\tilde{D}^{\prime}, \tilde{k}: U \subset \tilde{P}-\tilde{D}^{\prime}$ and $\tilde{k}^{\prime}: \tilde{P}-\tilde{D}^{\prime} \subset \tilde{P}$ have corresponding meanings, then we have a spectral sequence whose $E_{1}$-term is the same as in the constant case, but whose differentials are different:

$$
E_{1}^{p, q}=H^{q}\left(\tilde{P}, \Omega_{\tilde{P}}^{p}(\log \tilde{D})\right) \Rightarrow H^{p+q}\left(R^{\bullet} \tilde{k}_{!}^{\prime} R^{\bullet} \tilde{k}_{*} \mathbb{F}_{\eta}\right)
$$

From the preceding calculation we deduce that this sequence degenerates at the $E_{2^{-}}$ term and that $E_{2}^{0, p}=H^{p}\left(A^{\bullet}, \eta \wedge\right)=H^{p}\left(R^{\bullet} \tilde{k}_{1}^{\prime} R^{\bullet} \tilde{k}_{*} \mathbb{F}_{\eta}\right)$. The image $D^{\prime}$ of $\tilde{D}^{\prime}$ in $P$ is the union of abnormal facets $L$ for which $E_{L}$ is a positive integer; if $k: U \subset P-D^{\prime}$ and $k^{\prime}: P-D^{\prime} \subset P$ are the inclusions, then $H^{p}\left(R \tilde{k}_{!}^{\prime} R \tilde{k}_{*} \mathbb{F}_{\eta}\right) \cong H^{p}\left(R k_{1}^{\prime} R \tilde{k}_{*} \mathbb{F}_{\eta}\right) \cong$ $H_{\Phi(E)}^{p}\left(U ; \mathbb{F}_{\eta}\right)$.

(4.3) We now return to the affine case and assume that $P$ arises from a projective completion of an affine space $V$.

Given $L \in \mathcal{C}^{p}$, set

$$
\tilde{s}_{\eta}(L):=\sum_{H \supset L, H \in \mathcal{C}} \operatorname{Res}_{H}(\eta) \omega_{H} \in A^{1} \otimes \mathbb{C}
$$

and define for a flag $L^{\bullet}$ of positive length

$$
\tilde{s}_{\eta}\left(L^{\bullet}\right)=\tilde{s}_{\eta}\left(L^{1}\right) \wedge \tilde{s}_{\eta}\left(L^{2}\right) \wedge \cdots \wedge \tilde{s}_{\eta}\left(L^{p}\right) \in A^{p} \otimes \mathbb{C}
$$

We complete this definition by putting $\tilde{s}_{\eta}\left(L^{0}\right)=1$. One verifies easily that $\tilde{s}_{\eta}$ factors through a homomorphism $s_{\eta}: F^{p} \rightarrow A^{p} \otimes \mathbb{C}$ and that this factor is a chain map

$$
s_{\eta}:\left(F^{\bullet}, d\right) \rightarrow\left(A^{\bullet} \otimes \mathbb{C}, \eta \wedge\right) .
$$

So $s_{\eta}$ induces a homomorphism $H^{\bullet}(V, D ; \mathbb{Z}) \rightarrow H_{\Phi(\eta)}^{\bullet}\left(U ; \mathbb{F}_{\eta}\right)$. It is desirable to have a reasonable topological interpretation of this map, especially for its image.

If we identify $A^{\bullet}$ with the dual of $F^{\bullet}$, we end up with a bilinear form

$$
S_{\eta}: F^{p} \otimes F^{p} \rightarrow \mathbb{C}
$$

Concretely, if $L^{\bullet}$ and $L^{\prime}$ are $p$-flags, then

$$
S_{\eta}\left(L^{\bullet}, L^{\prime \bullet}\right)=\sum_{\left(H_{1}, \ldots, H_{p}\right), \sigma} \operatorname{sign}(\sigma) \eta_{H_{1}} \cdots \eta_{H_{p}},
$$

where the sum is over all ordered $p$-tuples $\left(H_{1}, \ldots, H_{p}\right)$ in $\mathcal{C}$ and $\sigma \in \mathfrak{S}_{p}$ with the property that $L^{k}=H_{1} \cap \cdots \cap H_{k}$ and $L^{\prime k}=H_{\sigma(1)} \cap \cdots \cap H_{\sigma(k)}$ for $k=1, \cdots, p$. (Notice that given $\left(H_{1}, \ldots, H_{p}\right)$, there can be at most one $\sigma \in \mathfrak{S}_{p}$ with these properties, so that the sum is really over a set of $p$-tuples in $\mathcal{C}$.) From this formula we see that $S_{\eta}$ is symmetric. 


\section{Higher DiRECT images OF A KZ SYSTEM}

(5.1) We continue with the situation of the previous section. In addition we assume given a linear subspace $T$ of the translation space of $V$. We denote the quotient affine space $T \backslash V$ by $V^{\prime}$ and the projection map $V \rightarrow V^{\prime}$ by $\pi$. The members of $\mathcal{C}$ that are $T$-invariant define a a collection $\mathcal{C}^{\prime}$ of hyperplanes of $W$. We shall make the assumption that every facet of $\mathcal{C}$ projects onto a facet of $\mathcal{C}^{\prime}$; this assumption can always be made to hold by adding to $\mathcal{C}^{\prime}$ the hyperplanes that are images of facets of $\mathcal{C}$.

We have a corresponding algebra $A^{\prime \bullet}$ of logarithmic forms which we often regard as a subalgebra of $A^{\bullet}$. We form the algebra of relative forms:

$$
A_{\pi}^{\bullet}:=A^{\bullet} /\left(A^{\prime 1} \wedge A^{\bullet-1}\right) \text {. }
$$

Lemma. The sequence

$$
0 \rightarrow A^{\prime 1} \otimes\left(A_{\pi}^{\bullet-1}, \eta \wedge\right) \stackrel{\wedge}{\longrightarrow}\left(A^{\bullet}, \eta \wedge\right) \rightarrow\left(A_{\pi}^{\bullet}, \eta \wedge\right) \rightarrow 0
$$

is a short exact sequence of complexes.

Proof (sketch). We have to show that the map $\wedge: A^{\prime 1} \otimes A_{\pi}^{\bullet-1} \rightarrow A^{\prime 1} \wedge A_{\pi}^{\bullet-1}$ is injective. So let $\sum_{H \in \mathcal{C}^{\prime}} \omega_{H} \wedge \zeta_{H}=0$, with $\zeta_{H} \in A^{p}$. Fix $H_{0} \in \mathcal{C}^{\prime}$. Taking the residue along $H_{0}$ yields an an identity

$$
\zeta_{H_{0}} \mid H_{0}-\sum_{H \in \mathcal{C}^{\prime}-H_{0}} \omega_{H} \wedge \operatorname{Res}_{H_{0}} \zeta_{H}=0 .
$$

For $H \neq H_{0}, \operatorname{Res}_{H_{0}} \zeta_{H}$ is the restriction of some $\xi_{H} \in A^{p-1}$. The difference of $\zeta_{H_{0}}$ and $\sum_{H \in \mathcal{C}^{\prime}-\left\{H_{0}\right\}} \omega_{H} \wedge \xi_{H}$ is then an element of $A^{p}$ with trivial restriction to $H_{0}$. It is then not hard to show that this expression is in the ideal of $A^{\bullet}$ spanned by the $\omega_{H}$ with $H$ parallel to $H_{0}$. In particular, $\zeta_{H_{0}} \in A^{\prime 1} \wedge A^{p-1}$.

The long exact cohomology for this short exact sequence has a differential

$$
H^{p}\left(A_{\pi}^{\bullet}, \eta \wedge\right) \rightarrow A^{\prime 1} \otimes H^{p}\left(A_{\pi}^{\bullet}, \eta \wedge\right),
$$

which, when regarded as an element of $A^{\prime 1} \otimes \operatorname{End}\left(H^{p}\left(A_{\pi}^{\bullet}\right)\right)$, is denoted by $E_{\eta}$. Since the square of the differential is zero, we have $E_{\eta} \wedge E_{\eta}=0$, so that $E_{\eta}$ defines a KZ system; we will refer to this as the direct image $K Z$ system defined by $\eta$.

The underlying local system has a topological interpretation. For this we projectively complete $V$ in the $T$-direction. This gives a (trivial) bundle of projective spaces $\tilde{\pi}: \tilde{V} \rightarrow V^{\prime}$. The closures of the members of $\mathcal{C}$ plus the $\mathbb{P}(T)$-bundle at infinity define a "projective arrangement $\tilde{\mathcal{C}}$ over $V^{\prime}$ ".

Much of what we did in the absolute case carries over this relative case: Let $U^{\prime}$ denote the complement of the union of the members of $\mathcal{C}^{\prime}$ in $V^{\prime}$ and let $\mathbb{H}_{\eta}^{p}$ denote the sheaf on $U^{\prime}$ associated to the presheaf which assigns to every open $B \subset U^{\prime \prime}$ the cohomology group $H_{\Phi(\eta) \mid \pi^{-1} B \cap U}^{p}\left(\pi^{-1} B \cap U ; \mathbb{F}_{\eta}\right)$. This sheaf is locally constant and the underlying holomorphic vector bundle $\mathcal{O}_{U^{\prime}} \otimes \mathbb{H}_{\eta}^{p}$ has the following algebraic description: Let $\mathcal{A}_{\pi}^{p}$ denote the sheaf on $U^{\prime}$ of relative logarithmic $p$-forms; (so that a section of $\mathcal{A}_{\pi}^{p}$ over $B$ is a relative meromorphic $p$-form on $\tilde{\pi}^{-1} B$, with logarithmic poles along the members of $\tilde{\mathcal{C}}$ ). Both the exterior derivative and wedging with $\eta$ makes $\mathcal{A}_{\pi}^{\bullet}$ a complex of sheaves. The two operations anti-commute and so we have a complex $\left(\mathcal{A}_{\pi}^{\bullet}, d+\eta \wedge\right)$. 
(5.2) Proposition. There is a natural isomorphism

$$
\mathcal{H}^{p}\left(\mathcal{A}_{\pi}^{\bullet}, d+\eta \wedge\right) \cong \mathcal{O}_{U^{\prime}} \otimes \mathbb{H}_{\eta}^{p}
$$

The proof is similar to that of (4.2).

Notice that every element of $A_{\pi}^{\bullet}$ defines a global section of $\mathcal{A}_{\pi}^{\bullet}$. This restriction homomorphism

$$
r^{\bullet}:\left(A_{\pi}^{\bullet}, \eta \wedge\right) \rightarrow\left(H^{0}\left(U^{\prime} ; \mathcal{A}_{\pi}^{\bullet}\right), d+\eta \wedge\right)
$$

is a morphism of complexes and so induces homomorphisms

$$
H^{p}\left(r^{\bullet}\right): H^{p}\left(A_{\pi}^{\bullet}, \eta \wedge\right) \rightarrow H^{0}\left(U^{\prime} ; \mathcal{H}^{p}\left(\mathcal{A}_{\pi}^{\bullet}, d+\eta \wedge\right)\right) \cong H^{0}\left(U^{\prime} ; \mathcal{O}_{U^{\prime}} \otimes \mathbb{H}_{\eta}^{p}\right)
$$

(5.3) Proposition. The maps $r^{\bullet}$ and $H^{p}\left(r^{\bullet}\right)$ are injections.

Proof (sketch). Let $\zeta \in A^{p}$ map to an element of the kernel of $r^{p}$. This implies that for every $y \in U^{\prime \prime}$, the restriction of $\eta$ to the fiber $\tilde{V}_{y}$ is exact. This restriction is a logarithmic form on a projective variety. According to Deligne [3] such a form can be exact only when it is zero. So locally over $U^{\prime}, \zeta$ is in the ideal generated by $A^{\prime 1}$. A computation shows that this this even true locally over all of $V^{\prime}$. Then $\eta$ can be written as $\sum_{H \in \mathcal{C}^{\prime}} \omega_{H} \wedge \zeta_{H}$ with $\zeta_{H}$ a linear combination of elements of $A^{p-1}$ with coefficients polynomial functions on $V^{\prime}$. If we replace these polynomial functions by their constant term then the corresponding linear combination is still equal to $\zeta$ (we are in effect taking the degree zero part of an equation), and so $\zeta \in A^{\prime 1} \wedge A^{p-1}$. This shows that $r^{\bullet}$ is injective. For the injectivity of $H^{p}\left(r^{\bullet}\right)$ one proceeds in the same way: if $\zeta \in A^{p}$ can be written as $\eta \wedge \xi+\sum_{H \in \mathcal{C}^{\prime}} \omega_{H} \wedge \zeta_{H}$ with $\xi$ and $\zeta_{H}$ linear combinations of elements of $A^{p-1}$ with polynomial functions on $V^{\prime}$ as coefficients, then by taking the degree zero part we find a solution inside $A^{p-1}$ itself.

Note that in this discussion the residues of $\eta$ along the members of $\mathcal{C}^{\prime}$ do not matter.

It is likely that a weak Lefschetz theorem holds which asserts that $H^{p}\left(r^{\bullet}\right)$ is an isomorphism onto $H^{0}\left(U^{\prime} ; \mathcal{H}_{\eta}^{p}\right)$ for $p<\operatorname{dim} T$.

(5.4) Proposition. The natural flat connection on $\mathcal{O}_{U^{\prime}} \otimes \mathbb{H}_{\eta}^{p}$ induces the $K Z$ connection $E_{\eta}$ on $H^{p}\left(A_{\pi}^{\bullet}, \eta\right)$.

Proof. The flat connection $\mathcal{O}_{U^{\prime}} \otimes \mathbb{H}_{\eta}^{p}$ transported to $\mathcal{H}^{p}\left(\mathcal{A}_{\pi}^{\bullet}, d+\eta \wedge\right)$ is given by a map

$$
D: \mathcal{H}^{p}\left(\mathcal{A}_{\pi}^{\bullet}, d+\eta \wedge\right) \rightarrow \Omega_{U^{\prime}}^{1} \otimes \mathcal{H}^{p}\left(\mathcal{A}_{\pi}^{\bullet}, d+\eta \wedge\right)
$$

which can be described as follows (see for instance [2]): a section of $\mathcal{H}^{p}\left(\mathcal{A}_{\pi}^{\bullet}, d+\right.$ $\eta \wedge)$ over a Stein coordinate patch $\left(B ; z_{1}, z_{2}, \ldots, z_{k}\right)$ of $U^{\prime}$ is representable by a logarithmic form $\zeta$ on $\tilde{\pi}^{-1} B$ with the property that $d \zeta+\eta \wedge \zeta=\sum_{i=1}^{k} d z_{i} \wedge \zeta_{i}$ for certain logarithmic forms $\zeta_{i}$ on $\tilde{\pi}^{-1} B$. Then $\zeta_{i}$ maps to a cocycle of $\left(\mathcal{A}_{\pi}^{p}, d+\eta \wedge\right)$ and

$$
D([\zeta])=\sum_{i=1}^{k} d z_{i} \otimes\left[\zeta_{i}\right] .
$$

The proposition now follows easily. 


\section{Embedding a LiE Algebra COMPlEX in A FLAG COMPLEX}

In this section $U_{r}$ is the free associative algebra over $\mathbb{C}$ on $r$ variables $f_{1}, \ldots, f_{r}$ and $L_{r} \subset U_{r}$ the (free) complex Lie algebra generated by these elements. The algebra $U_{r}$ is multigraded by $\mathbb{Z}_{\geq 0}^{r}$ and $L_{r}$ inherits this grading.

(6.1) Let $\tilde{V}_{r}$ be a complex vector space of dimension $r$ with coordinates $t_{1}, \ldots, t_{r}$. Put $z=-\sum_{i} t_{i}$, and let $V_{r}$ denote the quotient of $\tilde{V}_{r}$ by the line $t_{1}=\cdots=t_{r}$. Let $\tilde{\mathcal{C}}_{r}$ be the collection of hyperplanes in $\tilde{V}$ having an equation $z=t_{i}$ or $t_{i}=t_{j}$, and $\mathcal{C}_{r}$ those of $V_{r}$ having an equation by $t_{i}=t_{j}$. We identify $p$-flags of $\mathcal{C}_{r}$ with $p$-flags of $\tilde{\mathcal{C}}_{r}$ so that $F^{\bullet}\left(\mathcal{C}_{r}\right)$ can be regarded as a graded submodule of $F^{\bullet}\left(\tilde{\mathcal{C}}_{r}\right)$. For a facet $L$ of $\tilde{\mathcal{C}}_{r}$ and $i \in\{1, \ldots r\}$, define $\epsilon(i, L)$ to be 1 resp. -1 if the number of hyperplanes of the form $z=t_{j}, j>i$ which contain $L$ is even resp. odd. We define an action of $U_{r}$ on $F^{\bullet}\left(\hat{\mathcal{C}}_{r}\right)$ by letting $f_{i}$ act as "left multiplication" with $\left\{z=t_{i}\right\}$ up to sign:

$$
f_{i}\left[L^{p} \subset \cdots \subset L^{0}\right]:=\left\{\begin{array}{l}
0 \quad \text { if } L^{p} \subset\left(z=t_{i}\right) \\
\epsilon\left(i, L^{p}\right)\left[\left(z=t_{i}\right) \cap L^{p} \subset L^{p} \subset \cdots \subset L^{0}\right] \text { else. }
\end{array}\right.
$$

Then we have a linear mapping

$$
\tilde{\psi}: U_{r} \rightarrow F^{\bullet}\left(\tilde{\mathcal{C}}_{r}\right), \quad u \mapsto u\left[L_{0}\right]
$$

which preserves the (total) degree. Notice that for $i>j,\left[f_{i}, f_{j}\right]$ is left multiplication with $-\left[\left(z=t_{i}=t_{j}\right) \subset\left(t_{i}=t_{j}\right)\right]$. With induction we see that a $k$-fold commutator $\left[f_{i_{k+1}},\left[f_{i_{k}},\left[\cdots, f_{i_{1}}\right] \cdots\right]\right.$ with $i_{k+1}>i_{k}>\cdots>i_{1}$ acts as left multiplication with $(-1)^{k}$ times the $(k+1)$-flag

$\left[\left(z=t_{i_{k+1}}=\cdots=t_{i_{1}}\right) \subset\left(t_{i_{k+1}}=\cdots=t_{i_{1}}\right) \subset\left(t_{i_{k}}=\cdots=t_{i_{1}}\right) \subset \cdots \subset\left(t_{i_{2}}=t_{i_{1}}\right)\right]$,

and a permutation $\sigma$ of these elements has the effect of multiplying the flag by the sign of $\sigma$. So if we omit the smallest term we obtain an element of $F^{k}\left(\mathcal{C}_{r}\right)$. This defines a linear map

$$
\psi: L_{r} \rightarrow F^{\bullet}\left(\mathcal{C}_{r}\right) \otimes \mathbb{C}
$$

of degree -1 .

(6.2) Let $V_{r, n}$ be a complex vector space of dimension $r+n-1$ and $\left\{z_{1}, \ldots, z_{n}\right.$; $\left.t_{1}, \ldots, t_{r}\right\}$ a set of generators of its dual whose sum is 0 . Let $\mathcal{C}_{r, n}$ be the collection of hyperplanes of the form $z_{\nu}=t_{i}$ and $t_{i}=t_{j}$. There is an obvious map $\pi_{r, n}$ : $V_{r, n} \rightarrow V_{r}$ and a corresponding embed $\operatorname{ding} \mathcal{C}_{r} \subset \mathcal{C}_{r, n}$ and consequently a linear map $\psi: L_{r} \rightarrow F^{r-p}\left(\mathcal{C}_{r, n}\right) \otimes \mathbb{C}$. Substituting $z_{\nu}$ for $z$ defines an embedding $\tilde{\mathcal{C}}_{r} \subset \mathcal{C}_{r, n}$ and hence a linear map $\tilde{\psi}_{\nu}: U_{r} \rightarrow F^{\bullet}\left(\mathcal{C}_{r, n}\right) \otimes \mathbb{C}$. Now consider the linear map

$$
\left(L_{r}^{\otimes p} \otimes U_{r}^{\otimes n}\right)_{(1,1, \ldots, 1)} \rightarrow F^{r-p}\left(\mathcal{C}_{r, n}\right) \otimes \mathbb{C}
$$

defined by the rule

$$
g_{p} \otimes \cdots \otimes g_{1} \otimes u_{1} \otimes \cdots \otimes u_{n} \mapsto \psi\left(g_{p}\right) \cdots \psi\left(g_{1}\right) \tilde{\psi}_{1}\left(u_{1}\right) \cdots \tilde{\psi}_{n}\left(u_{n}\right) .
$$


If the $g_{i}^{\prime} s$ are homogeneous, then interchanging $g_{i}$ and $g_{i+1}$ makes the image acquire the $\operatorname{sign}(-1)^{\left(\operatorname{deg}\left(g_{i}\right)-1\right)\left(\operatorname{deg}\left(g_{i+1}\right)-1\right)}$. We wish to modify the definition of this mapping so that this change in sign becomes -1 instead, thus making the modified map factorize through $\left(\wedge^{p} L_{r} \otimes U_{r}^{\otimes n}\right)_{(1,1, \ldots, 1)}$. To this end, assume that $g_{p}, \ldots, g_{1}$ resp. $u_{1}, \ldots, u_{n}$ are Lie-monomials resp. noncommutative monomials in $f_{1}, \ldots, f_{r}$. If $\left(g_{p} \otimes \cdots \otimes g_{1} \otimes u_{1} \otimes \cdots \otimes u_{n}\right)$ has multidegree $(1, \ldots, 1)$ and $f_{1}, \ldots f_{n}$ appear in the order $f_{\sigma(r)}, \ldots, f_{\sigma(1)}$, then we multiply the above expression with $\operatorname{sign}(\sigma)(-1)^{\sum_{i} i\left(\operatorname{deg}\left(g_{i}\right)-1\right)}$. This has indeed the desired effect with respect to interchanging adjacent $g_{i}$ 's so that we now have a well-defined map

$$
\Psi_{r, n}:\left(\wedge^{\bullet} L_{r} \otimes U_{r}^{\otimes n}\right)_{(1,1, \ldots, 1)} \rightarrow F^{r-\bullet}\left(\mathcal{C}_{r, n}\right) \otimes \mathbb{C}
$$

(6.3) Claim. This is a $\mathfrak{S}_{r}$-equivariant isomorphism of graded vector spaces.

Proof. This is left to the reader. For equivariance, one checks that $\Psi_{r, n}$ commutes with interchanging the indices $i$ and $i+1$.

Recall that for any $L_{r}$-module $M$ one has the standard chain complex

$$
C \cdot\left(L_{r}, M\right)=\left(\wedge^{\bullet} L_{r} \otimes M, \partial\right),
$$

whose differential is defined by

$$
\begin{aligned}
\partial\left(g_{p} \wedge\right. & \left.\cdots \wedge g_{1} \otimes m\right)=\sum_{i=1}^{p}(-1)^{i-1} g_{p} \wedge \cdots \wedge \widehat{g_{i}} \wedge \cdots \wedge g_{1} \otimes g_{i} m \\
& +\sum_{1 \leq i<j \leq p}^{p}(-1)^{i+j} g_{p} \wedge \cdots \wedge \widehat{g_{j}} \wedge \cdots \wedge \widehat{g_{i}} \wedge \cdots \wedge g_{1} \wedge\left[g_{j}, g_{i}\right] \otimes m
\end{aligned}
$$

If $M$ is multigraded by $\mathbb{Z}_{>0}^{r}$ in a way that is compatible with the $L_{r}$-action (think of $U_{r}^{\otimes n}$ ), then the differential respects the multigrading of the complex. So both source and target of $\Psi_{r, n}$ are complexes. One can check that $\Psi_{r, n}$ commutes with the differentials. The proof is a matter of careful bookkeeping of the signs and so we omit it. Thus:

(6.4) Proposition. We have an $\mathfrak{S}_{r}$-equivariant isomorphism of complexes

$$
\Psi_{r, n}:\left(C \cdot\left(L_{r}, U_{r}^{\otimes n}\right)_{(1,1, \ldots, 1)} \rightarrow F^{r-\bullet}\left(\mathcal{C}_{r, n}\right) \otimes \mathbb{C} .\right.
$$

With this isomorphism constructed, we can identify the other multigraded pieces of $C .\left(L_{r}, U_{r}^{\otimes n}\right)$ with flag complexes as well. Given $\lambda=\left(\lambda_{1}, \ldots, \lambda_{r}\right) \in \mathbb{Z}_{\geq 0}^{r}$, let $U_{\lambda}$ be the free associative algebra on generators $f_{i, j}, i=1, \ldots, r, j=1, \ldots, \lambda_{i}$. We define an embedding $U_{r} \rightarrow U_{\lambda}$ by mapping the generator $f_{i}$ to $\sum_{j=1}^{\lambda_{i}} f_{i, j}$. This identifies $U_{r}$ with the part of $U_{\lambda}$ that is fixed under the obvious action of $\mathfrak{S}_{\lambda}:=\mathfrak{S}_{\lambda_{1}} \times \cdots \times \mathfrak{S}_{\lambda_{r}}$. Similarly, we have an isomorphism

$$
\left(C \cdot ( L _ { r } , U _ { r } ^ { \otimes n } ) _ { \lambda } \cong \left(C \cdot\left(L_{\lambda}, U_{\lambda}^{\otimes n}\right)_{(1,1, \ldots, 1)}^{\mathfrak{S}_{\lambda}}\right.\right.
$$

In view of (6.4) and the results of section 3 we get: 
(6.5) Theorem. For every multidegree $\lambda \in \mathbb{Z}_{\geq 0}^{r}$ we have a natural isomorphism of complexes

$$
\Psi_{r, \lambda}:\left(C \cdot\left(L_{r}, U_{r}^{\otimes n}\right)_{\lambda} \cong F^{|\lambda|-\bullet}\left(\mathcal{C}_{\lambda, n}\right)^{\mathfrak{S}_{\lambda}} \otimes \mathbb{C},\right.
$$

(where $\mathcal{C}_{\lambda, n}$ has the obvious interpretation) and (dually) an isomorphism of complexes

$$
\Psi_{r, \lambda}^{\vee}: A^{\bullet}\left(\mathcal{C}_{\lambda, n}\right)^{\mathfrak{S}_{\lambda}} \otimes \mathbb{C} \cong\left(C^{\bullet}\left(L_{r},\left(\left(U_{r}\right)^{\vee}\right)^{\otimes n}\right)_{-\lambda}\right.
$$

The preceding construction is somewhat unsatisfactory since it appears to come out of the blue. One would like to see a more conceptual interpretation of this isomorphism.

\section{A KZ System ATTACHED TO A Class OF LIE ALgEBRA'S}

We begin by briefly reviewing the beginnings of the theory of Kac-Moody Lie algebra's (in fact, of an even more general class of Lie algebra's). The reader will find more information in the book by Kac [5].

First a remark concerning graded duals: if $V=\oplus_{a \in A} V_{a}$ is a vector space graded by an abelian group $A$, then its graded dual $V^{\vee}$ is defined as the direct sum of the duals of its homogeneous components with the understanding that the dual of $V_{a}$ has degree $-a$. So if the homogeneous components $V_{a}$ are finite dimensional, then the double graded dual of $V$ is canonically isomorphic to $V$.

(7.1) We start out with a finite dimensional complex vector space $\mathfrak{h}$, a nondegenerate symmetric bilinear form $B$ on $\mathfrak{h}$ and linearly independent forms $\alpha_{1}, \ldots, \alpha_{r} \in$ $\mathfrak{h}^{\vee}$ (called the fundamental roots). Let $h_{i} \in \mathfrak{h}$ be characterized by the property that $B\left(h_{i}, h\right)=\alpha_{i}(h)$ for all $h \in \mathfrak{h}$ and let $\tilde{\mathfrak{g}}$ be the Lie algebra generated by $\mathfrak{h}$ and indeterminates $e_{1}^{+}, \ldots e_{r}^{+}, e_{1}^{-}, \ldots e_{r}^{-}$subject to the relations

$$
\begin{aligned}
{\left[e_{i}^{+}, e_{j}^{-}\right] } & =\delta_{i j} h_{i}, \\
{\left[h, e_{i}^{\epsilon}\right] } & =\epsilon \alpha_{i}(h) e_{i}^{\epsilon}, \\
{\left[h, h^{\prime}\right] } & =0
\end{aligned}
$$

for all $h, h^{\prime} \in \mathfrak{h}$ and $\epsilon \in\{ \pm\}$. The elements $e_{1}^{\epsilon}, \ldots, e_{n}^{\epsilon}$ generate a free Lie subalgebra $\tilde{\mathfrak{n}}_{\epsilon}$ and we have a triangular decomposition

$$
\tilde{\mathfrak{g}}=\tilde{\mathfrak{n}}_{-} \oplus \mathfrak{h} \oplus \tilde{\mathfrak{n}}_{+}
$$

The adjoint action of $\mathfrak{h}$ on $\tilde{\mathfrak{g}}$ is semi-simple. Clearly, the weights that occur in $\mathfrak{n}_{-}$ resp. $\mathfrak{n}_{+}$are negative resp. positive integral linear combinations of the $\alpha_{i}$ 's so that the triangular decomposition is refined by the weight decomposition

$$
\tilde{\mathfrak{g}}=\oplus_{\lambda} \tilde{\mathfrak{g}}_{\lambda} .
$$

Notice that if we replace $B$ by a nonzero scalar multiple we get an isomorphic Lie algebra.

(7.2) The form $B$ extends (uniquely) to $\tilde{\mathfrak{g}}$ as a $\tilde{\mathfrak{g}}$-invariant symmetric bilinear form $\tilde{B}$. In particular, $\tilde{\mathfrak{g}}_{\lambda}$ and $\tilde{\mathfrak{g}}_{\mu}$ are perpendicular unless $\lambda+\mu=0$. One checks 
easily that $\tilde{B}\left(e_{i}^{+}, e_{j}^{-}\right)=\delta_{i j}$. The Chevalley involution $\tilde{\tau}: \hat{\mathfrak{g}} \rightarrow \tilde{\mathfrak{g}}$ is defined as minus the identity on $\mathfrak{h}$ and sends $e_{i}^{ \pm}$to $-e_{i}^{\mp}$. So $\tilde{\tau}$ interchanges $\mathfrak{g}_{\lambda}$ and $\mathfrak{g}_{-\lambda}$. It is symmetric with respect to $\tilde{B}$, so that

$$
\tilde{S}\left(g, g^{\prime}\right):=-\tilde{B}\left(\tilde{\tau} g, g^{\prime}\right)
$$

is a symmetric bilinear form on $\tilde{\mathfrak{g}}$. Now the weight decomposition $\tilde{\mathfrak{g}}=\oplus_{\lambda} \tilde{\mathfrak{g}}_{\lambda}$ is orthogonal with respect to $\tilde{S}$. Observe that $\tilde{S}$ is not $\mathfrak{g}$-invariant but satisfies

$$
\tilde{S}\left(\left[g, g_{1}\right], g_{2}\right)+\tilde{S}\left(g_{1},\left[\tilde{\tau}(g), g_{2}\right]\right)=0 \text {. }
$$

We put

$$
\tilde{\mathfrak{b}}_{+}:=\mathfrak{h} \oplus \tilde{\mathfrak{n}}_{+}
$$

The nilspace of $\tilde{S}$ is also the nilspace of $\tilde{B}$ and is therefore a graded ideal. The quotient algebra, denoted $\mathfrak{g}$, inherits the grading of $\mathfrak{\mathfrak { g }}$ :

$$
\mathfrak{g}:=\oplus{ }_{\lambda} \mathfrak{g}_{\lambda},
$$

the forms $\tilde{B}$ resp. $\tilde{S}$ descend to a nondegenerate forms $B$ resp. $S$ on $\mathfrak{g}$ and $\tilde{\tau}$ induces an involution $\tau$ of $\mathfrak{g}$. The grading is orthogonal with respect $S$ and the triangular decomposition of $\tilde{\mathfrak{g}}$ determines one of $\mathfrak{g}$ :

$$
\mathfrak{g}:=\mathfrak{n}_{-} \oplus \mathfrak{h} \oplus \mathfrak{n}_{-} .
$$

Notice that $B$ induces a pairing $\mathfrak{n}_{-} \times \mathfrak{n}_{+} \rightarrow \mathbb{C}$. This pairing is perfect in the graded sense, so that $\mathfrak{n}_{+}$can be identified with the graded dual of $\mathfrak{n}_{-}$.

Remark. This class of algebra's contains the Kac-Moody algebra's associated to a symmetrizable Cartan matrix, in particular it contains the finite dimensional complex reductive Lie algebra's. To see this, assume that $B\left(h_{i}, h_{i}\right) \neq 0$ for all $i$ and consider the matrix $\left(n_{i j}:=2 B\left(h_{i}, h_{j}\right) / B\left(h_{i}, h_{i}\right)\right)_{i, j}$. If this is a generalized Cartan matrix in the sense that its off-diagonal entries are nonnegative integers with $n_{i j}=0 \Leftrightarrow n_{j i}=0$, then $\mathfrak{g}$ is one of its associated Kac-Moody algebra's. This follows from a theorem of Gabber and Kac which states that in this situation the nilspace of $\tilde{S}$ is generated by the "Serre elements" $\left(\operatorname{ad} e_{i}^{\epsilon}\right)^{-n_{i j}+1}\left(e_{j}^{\epsilon}\right)(i \neq j)$. Every symmetrizable generalized Cartan matrix arises this way. If $\left(n_{i j}\right)$ is a Cartan matrix, then $\mathfrak{g}$ is a finite dimensional reductive Lie algebra and the image of $\mathfrak{h}$ in it is a Cartan subalgebra.

The projection $\tilde{\mathfrak{b}}_{+} \rightarrow \mathfrak{h}$ is a Lie algebra homomorphism and so every $\mu \in \mathfrak{h}^{*}$ defines a one-dimensional representation $\mathbb{C}_{\mu}$ of $\tilde{\mathfrak{b}}_{+}$. The induced module

$$
\tilde{M}(\mu):=U \tilde{\mathfrak{g}} \otimes_{U \tilde{\mathfrak{b}}_{+}} \mathbb{C}_{\mu}
$$

is called the Verma module of $\tilde{\mathfrak{g}}$ with highest weight $\mu$. Since we have a factorization $U \tilde{\mathfrak{g}}=U \tilde{\mathfrak{n}}_{-} \otimes U \tilde{\mathfrak{b}}_{+}, M(\mu)$ is canonically isomorphic to $U\left(\tilde{\mathfrak{n}}_{-}\right)$as a $\tilde{\mathfrak{n}}_{-- \text {-module. Notice }}$ 
that $U\left(\tilde{\mathfrak{n}}_{-}\right)$is a free associative algebra on $e_{1}^{-}, \ldots, e_{r}^{-}$. Since $\mathfrak{h}$ acts semi-simply on $U \mathfrak{g}$, it does so on $\tilde{M}(\mu)$. Whence an eigen space decomposition:

$$
\tilde{M}(\mu)=\oplus_{\pi \in \mathfrak{h}^{*}} \tilde{M}(\mu)_{\pi}
$$

with $\tilde{M}(\mu)_{\pi}=0$ unless $\pi \in \mu+\mathbb{Z}_{\leq 0} \alpha_{1}+\cdots \mathbb{Z}_{\leq 0} \alpha_{r}$. We equip $M(\mu)$ with a bilinear form, also denoted by $\tilde{S}$, which is characterized by the property that $\tilde{S}(1 \otimes 1,1 \otimes 1)=$ 1 and $\tilde{S}(g x, y)+\tilde{S}(x, \tilde{\tau}(g) y)=0$ for all $g \in \mathfrak{g}$ and $x, y \in \tilde{M}(\mu)$. One verifies that $\tilde{S}$ is symmetric and that the grading of $\tilde{M}(\mu)$ is orthogonal with respect to $\tilde{S}$. There is an associated adjoint map

$$
\tilde{s}: \tilde{M}(\mu) \rightarrow \tilde{M}(\mu)^{\vee},
$$

which maps each homogeneous summand to its dual. If we twist the contragradient representation on $\tilde{M}(\mu)^{\vee}$ with the Chevalley involution, then this map becomes a $\tilde{\mathfrak{g}}$-homomorphism. The image of $\tilde{s}$ may be identified with the Verma module for $\mathfrak{g}$ with highest weight $\mu$ :

$$
M(\mu):=U \mathfrak{g} \otimes_{U \mathfrak{b}_{+}} \mathbb{C}_{\mu},
$$

in other words, $\tilde{s}$ induces a nondegenerate form $s$ on $M(\mu)$.

(7.3) We now fix weights $\mu_{1}, \ldots, \mu_{n} \in \mathfrak{h}^{*}$, and consider the $\tilde{\mathfrak{g}}$-module

$$
\tilde{M}:=\tilde{M}\left(\mu_{1}\right) \otimes_{\mathbb{C}} \cdots \otimes_{\mathbb{C}} \tilde{M}\left(\mu_{n}\right) .
$$

We restrict the module structure to $\tilde{\mathfrak{n}}_{-}$and form the standard chain complex $\left(\wedge^{\bullet} \tilde{\mathfrak{n}}_{-} \otimes \tilde{M}, \partial\right)$. The symmetric bilinear forms $\tilde{S}$ defined on $\tilde{n}_{-}$and the $\tilde{M}\left(\mu_{i}\right)^{\prime}$ s combine to give such a form (also denoted by $\tilde{S}$ ) on $\wedge^{\bullet} \tilde{\mathfrak{n}}_{-} \otimes \tilde{M}$. The associated adjoint map is

$$
\tilde{s}: \wedge^{\bullet} \tilde{\mathfrak{n}}_{-} \otimes \tilde{M} \rightarrow\left(\wedge^{\bullet} \tilde{\mathfrak{n}}_{-} \otimes \tilde{M}\right)^{\vee} .
$$

(7.4) Lemma. The image of $\tilde{s}: \wedge^{\bullet} \tilde{\mathfrak{n}}_{-} \otimes \tilde{M} \rightarrow\left(\wedge^{\bullet} \tilde{\mathfrak{n}}_{-} \otimes \tilde{M}\right)^{\vee}$ is a quotient complex of of $\left(\wedge^{\bullet} \tilde{\mathfrak{n}}_{-} \otimes \tilde{M}, \partial\right)$ whose graded dual is canonically isomorphic to the standard cochain complex of $\mathfrak{n}_{+}$with values in $M, \operatorname{Hom}\left(\wedge^{\bullet} \mathfrak{n}_{+}, M\right)$. This isomorphism multiplies the weights with -1 . In particular, the weight $\lambda$ piece of the pth homology group of the image of $\wedge^{\bullet} \tilde{s} \otimes s$ is dual to the weight $\lambda$ piece of $H^{p}\left(\mathfrak{n}_{+}, M\right)$.

Proof. From the preceding discussion it is clear that $\tilde{s}$ factorizes as

$$
\wedge^{\bullet} \tilde{\mathfrak{n}}_{-} \otimes \tilde{M} \rightarrow \wedge^{\bullet} \mathfrak{n}_{-} \otimes M \cong \wedge^{\bullet} \tilde{\mathfrak{n}}_{-}^{\vee} \otimes M^{\vee} \rightarrow\left(\wedge^{\bullet} \tilde{\mathfrak{n}}_{-} \otimes \tilde{M}\right)^{\vee}
$$

where the first map is surjective, the middle map is given by $s$ and the last one is injective. So the image can be identified with $\wedge^{\bullet} \mathfrak{n}_{-} \otimes M$, which is indeed a quotient complex of $\wedge \mathfrak{\mathfrak { n }}_{-} \otimes \tilde{M}$. Since $B$ induces a perfect pairing between $\mathfrak{n}_{-}$and $\mathfrak{n}_{+}$, the third term may be identified with $\wedge^{\bullet} \mathfrak{n}_{+} \otimes M^{\vee}$. The resulting isomorphism $\wedge \cdot \mathfrak{n}_{-} \otimes M \cong \wedge^{\bullet} \mathfrak{n}_{+} \otimes M^{\vee}$ is an isomorphism of complexes if we give $M^{\vee}$ the (untwisted) contragradient representation. Notice that this isomorphism multiplies the weights with -1 . The lemma follows from this. 
(7.5) Let $\alpha \in \mathfrak{h}^{*}$ be a root of $\mathfrak{h}$ in $\mathfrak{g}$, that is, a nonzero weight for which $\mathfrak{g}_{\lambda}$ is nontrivial. The form $B$ establishes a perfect pairing between the finite dimensional vector spaces $\mathfrak{g}_{\alpha}$ and $\mathfrak{g}_{-\alpha}$ and thus determines an element $B_{\alpha}^{\vee} \in \mathfrak{g}_{\alpha} \otimes \mathfrak{g}_{-\alpha}$. Since $B$ is symmetric, interchanging the factors transforms $B_{\alpha}^{\vee}$ in $B_{-\alpha}^{\vee}$. We denote by $B_{0}^{\vee} \in \mathfrak{h} \otimes \mathfrak{h}$ the symmetric tensor that represents the inverse of $B \mid \mathfrak{h} \times \mathfrak{h}$ and we put

$$
B^{\vee}:=B_{0}^{\vee}+\sum_{\alpha \text { a root }} B_{\alpha}^{\vee}
$$

considered as an element of a completed tensor product of $\mathfrak{g}$ with itself. In that sense it is $\mathfrak{g}$-invariant, just as in example 2 of (1.6) and for the same reason.

For $1 \leq k<l \leq n$, and $\alpha$ a root or zero, we let $\left(B_{\alpha}^{\vee}\right)_{k, l}$ denote the action of $B_{\alpha}^{\vee}$ on $M$ via the $k$ th and $l$ th factor. Notice that if this action is nontrivial, then $\alpha \in \mu_{k}+\mathbb{Z}_{\leq 0}\left\{\alpha_{1}, \ldots, \alpha_{r}\right\}$ and $-\alpha \in \mu_{l}+\mathbb{Z}_{\leq 0}\left\{\alpha_{1}, \ldots, \alpha_{r}\right\}$. So this is the case for only finitely many $\alpha$ 's. Therefore, there is a well-defined action of $B_{k, l}^{\vee}:=\sum_{\alpha}\left(B_{\alpha}^{\vee}\right)_{k, l}$ on $M$. By letting $\wedge^{\bullet} \mathfrak{n}_{+}$be inert, we extend this to an action on the standard cochain complex $\operatorname{Hom}\left(\wedge^{\bullet} \mathfrak{n}_{+}, M\right)$. Notice that $B_{k, l}^{\vee}$ is $\mathfrak{g}$-equivariant, respects the weights and commutes with the differential. So for every weight $\lambda \in \mathfrak{h}^{*}, B_{k, l}^{\vee}$ acts on $\operatorname{Hom}\left(\wedge^{\bullet} \mathfrak{n}_{+}, M\right)_{\lambda}$ and on $H^{p}\left(\mathfrak{n}_{+}, M\right)_{\lambda}$. Since $H^{0}\left(\mathfrak{n}_{+}, M\right)_{\lambda}$ can be identified with $\operatorname{Hom}_{\mathfrak{g}}(M(\lambda), M)$, this provides us with a generalization of example 2 of (1.6):

(7.6) Proposition. The quadruple $\left(V_{n}, \mathcal{C}_{n}, H^{p}\left(\mathfrak{n}_{+}, M\right)_{\lambda}, \sum_{1 \leq k<l \leq n} \omega_{k, l} \otimes B_{k, l}^{\vee}\right)$ defines a $K Z$ system.

The proof is similar. Our aim is to identify the $\mathrm{KZ}$ system defined by $H^{\bullet}\left(\mathfrak{n}_{+}, M\right)_{\lambda}$ with a summand of a direct image KZ system as discussed in section 5 . This we do in the next section.

\section{A KZ system as a Gauss-Manin system}

We continue with the situation of the previous section. We put $\mu:=\mu_{1}+\cdots+\mu_{n}$ and we fix a nonnegative integral weight $\lambda=\lambda_{1} \alpha_{1}+\cdots+\lambda_{r} \alpha_{r}, \lambda_{i} \in \mathbb{Z}_{\geq 0}$.

Define a logarithmic differential $\eta$ on $V_{\lambda, n}$ relative the collection $\mathcal{C}_{\lambda, n}$ by letting its residue along $\left(t_{i_{p}}=t_{j_{q}}\right)$ resp. $\left(z_{\nu}=t_{i_{p}}\right)$ be $B_{0}^{\vee}\left(\alpha_{i}, \alpha_{j}\right)$ resp. $-B_{0}^{\vee}\left(\mu_{\nu}, \alpha_{i_{p}}\right)$. According to (4.3) this defines a symmetric bilinear form $S_{\eta}$ on $F^{\bullet}\left(\mathcal{C}_{\lambda, n}\right)^{\mathfrak{S}_{\lambda}} \otimes \mathbb{C}$. Recall that $\tilde{\mathfrak{n}}_{-}$is a free Lie algebra on $r$ generators, and note that we have a $\tilde{\mathfrak{n}}_{--}$ module isomorphism $U \tilde{\mathfrak{n}}_{-} \cong \tilde{M}(\mu)$ of weight $\mu$. So (6.5) gives an isomorphism of complexes

$$
\Psi_{r, \lambda}: C \cdot\left(\tilde{n}_{-}, \tilde{M}\right)_{\mu-\lambda} \cong F^{|\lambda|-\bullet}\left(\mathcal{C}_{\lambda, n}\right)^{\mathfrak{S}_{\lambda}} \otimes \mathbb{C}
$$

(8.1) Proposition. The pull-back of $S_{\eta}$ under this isomorphism is $(-1)^{|\lambda|} S$.

The proof is straightforward and so we omit it.

(8.2) Corollary. The map $\Psi_{r, \lambda}$ induces an isomorphism of complexes between the image of $s_{\eta}$ (which is a subcomplex of $\left(A^{|\lambda|-\bullet}\left(\mathcal{C}_{\lambda, n}\right)^{\mathfrak{S}_{\lambda}} \otimes \mathbb{C}, \eta \wedge\right)$ ) and the dual of the Lie algebra cochain complex $\operatorname{Hom}\left(\wedge^{\bullet} \mathfrak{n}_{+}, M\right)$. In particular, we have isomorphisms

$$
H^{|\lambda|-p}\left(\operatorname{image}\left(s_{\eta}\right)\right) \cong\left(H^{p}\left(\mathfrak{n}_{+}, M\right)_{\mu-\lambda}\right)^{\vee}
$$


Proof. This follows immediately from the previous proposition, (4.3) and (6.5).

We add to the collection $\mathcal{C}_{\lambda, n}$ the hyperplanes $\left(z_{k}=z_{l}\right), k<l$. This arrangement is denoted $\mathcal{C}_{\lambda, n}^{*}$. The projection $\pi:\left(V_{\lambda, n}, \mathcal{C}_{\lambda, n}^{*}\right) \rightarrow\left(V_{n}, \mathcal{C}_{n}\right)$ brings us in the situation studied in section 5 . We use the notation of that section and so $A_{\pi}^{\bullet}$ will stand for the relative logarithmic differentials:

$$
A_{\pi}^{\bullet}:=A^{\bullet}\left(\mathcal{C}_{\lambda, n}^{*}\right) / A^{1}\left(\mathcal{C}_{n}\right) \wedge A^{\bullet-1}\left(\mathcal{C}_{\lambda, n}^{*}\right) \otimes \mathbb{C}
$$

There is an evident chain map

$$
\left(A^{|\lambda|-\bullet}\left(\mathcal{C}_{\lambda, n}\right) \otimes \mathbb{C}, \eta \wedge\right) \rightarrow\left(A_{\pi}^{\bullet}, \eta \wedge\right)
$$

In view of (8.2), we therefore have a natural map

$$
\psi:\left(H^{p}\left(\mathfrak{n}_{+}, M\right)_{\mu+\lambda}\right)^{\vee} \rightarrow H^{|\lambda|-p}\left(A_{\pi}^{\bullet}, \wedge \eta\right)^{\mathfrak{S}_{r}} .
$$

In (5.2) we found a $\mathrm{KZ}$ connection $E_{\eta}$ on $\left(V_{n}, \mathcal{C}_{n}\right)$ with values in the right hand side and in (7.6) we found a $\mathrm{KZ}$ connection $\sum_{1<k<l<n} \omega_{k, l} \otimes B_{k, l}^{\vee}$ on $\left(V_{n}, \mathcal{C}_{n}\right)$ with values in $\left.H^{p}\left(\mathfrak{n}_{+}, M\right)_{\mu+\lambda}\right)$ and hence also one with values in its dual. We twist the former by adding to $E_{\eta}$ the logarithmic form $\eta^{\prime}$ on $\left(V_{n}, \mathcal{C}_{n}\right)$ whose residue along $z_{k}=z_{l}, k<l$, is $B_{0}^{\vee}\left(\Lambda_{k}, \Lambda_{l}\right)$

(8.3) Theorem. The pull back of $E_{\eta}+\eta^{\prime}$ under $\psi$ is just the dual of the $K Z$ connection $\sum_{1<k<l<n} \omega_{k, l} \otimes B_{k, l}^{\vee}$ defined in (7.6). In particular, the $K Z$ connection on $U_{r}$ with values in $H^{|\lambda|}\left(A_{\pi}^{\bullet}, \wedge \eta\right)^{\mathfrak{S}_{r}}$ pulls back under $\psi$ to the $K Z$ connection with values $\operatorname{Hom}(M, M(\mu-\lambda))$.

We omit the proof. The point of this theorem is that it gives the KZ connection $\sum_{1 \leq k<l \leq n} \omega_{k, l} \otimes B_{k, l}^{\vee}$ with values in $\operatorname{Hom}(M, M(\mu-\lambda))$ a certain topological content.

\section{REFERENCES}

1. Deligne P., Equations differentielles à points réguliers singuliers, Lecture Notes in Math., vol. 163, Springer Verlag, Berlin and New York, 1970.

2. Deligne, Travaux de Griffiths, Sém. Bourbaki Exp. 376, Lecture Notes in Math., vol. 180, Springer Verlag, Berlin and New York, 1971.

3. P. Deligne, Théorie de Hodge II, Inst. Hautes Études Sci. Publ. Math. 40 (1971), 5-58; III, Inst. Hautes Études Sci. Publ. Math. 44 (1975), 6-77.

4. Esnault H., Schechtman V., Viehweg E., Cohomology of local sytems on the complement of hyperplanes, Invent. Math. 109 (1992), 557-661; Erratum, Invent. Math. 112 (1993), 447.

5. Kac V., Infinite dimensional Lie algebras, Cambridge University Press, Cambridge.

6. Knizhnik V., Zamolodchikov A., Current algebras and Wess-Zumino models in two dimensions, Nucl. Phys. B 247 (1984), 83-103.

7. Matsuo A., Integrable connections related to zonal spherical functions, Invent. Math. 110 (1992), 95-121.

8. Schechtman V.V., Varschenko A.N., Arrangements of hyperplanes and Lie algebra homology, Invent. Math. 106 (1991), 139-194. 